Mini Review

\section{Targeted and non-targeted effects of radiation in mammalian cells: An overview}

\author{
Rita Ghosh* and Surajit Hansda \\ Department of Biochemistry \& Biophysics, University of Kalyani, Kalyani-741235, West Bengal, \\ India
}

\section{Abstract}

Radiation of different wavelengths can kill living organisms, although, the mechanism of interactions differs depending on their energies. Understanding the interaction of radiation with living cells is important to assess their harmful effects and also to identify their therapeutic potential. Temporally, this interaction can be broadly divided in three stages - physical, chemical and biological. While radiation can affect all the important macromolecules of the cells, particularly important is the damage to its genetic material, the DNA. The consequences of irradiation include- DNA damage, mutation, cross-linkages with other molecules, chromosomal aberrations and DNA repair leading to altered gene expression and/or cell death. Mutations in DNA can lead to heritable changes and is important for the induction of cancer. While some of these effects are through direct interaction of radiation with the target, radiation can interact with the surrounding environment to result in its indirect actions. The effects of radiation depend not only on the total dose but also on the dose rate, LET etc. and also on the cell types. However, action of radiation on organisms is not restricted to interactions with irradiated cells, i.e. target cells alone; it also exerts non-targeted effects on neighboring unexposed cells to produce productive responses; this is known as bystander effect. The bystander effects of ionizing radiations are well documented and contribute largely to the relapse of cancer and secondary tumors after radiotherapy. Irradiation of cells with non-ionizing Ultra-Violet light also exhibits bystander responses, but such responses are very distinct from that produced by ionizing radiations.

\section{More Information}

*Address for Correspondence: Rita Ghosh, Professor, Department of Biochemistry \& Biophysics, University of Kalyani, Kalyani-741235, West Bengal, India, Tel: +919830489255; Email: rghosh_bcbp@klyuniv.ac.in; ritadg2001@yahoo.co.in

Submitted: March 15, 2021

Approved: April 09, 2021

Published: April 12, 2021

How to cite this article: Ghosh R, Hansda S. Targeted and non-targeted effects of radiation in mammalian cells: An overview. Arch Biotechnol Biomed. 2021; 5: 013-019.

DOI: 10.29328/journal.abb.1001023

Copyright: @ 2021 Ghosh R, et al. This is an open access article distributed under the Creative Commons Attribution License, which permits unrestricted use, distribution, and reproduction in any medium, provided the original work is properly cited.

Keywords: lonizing radiation; Non-ionizing radiation; Targeted action; non-targeted action; bystander effect

Check for updates

OPEN ACCESS
The term radiation relates to the transmission of energy by means of particles or waves. The biological effects of radiations were realized immediately after the discovery of X-rays by Roentgen in 1895 . The ability to photographically visualize the internal organs of the body, immediately opened up the possibilities of its use in medical diagnostics and soon its application in this area was realized within a year of its discovery [1]. However, it did not take long to realize the threat it poses to human health [2]. The realization of the potential of radiation to cause cell killing, also opened up yet a new avenue of therapeutics for cell proliferative diseases like cancer [3]. In spite of its vast prospective of application in biomedical arena, not much of serious research was undertaken until the 1950s, when radiation research emerged as a separate branch of science [4].

The biological effects of different ranges of electromagnetic spectrum is broadly clubbed according to their mode of interaction with matter, that depends on their energies, i.e., whether it is ionizing or non-ionizing radiation. Apart from the electromagnetic waves, particulate radiations too act as ionizing radiations, because of the high energy associated with them and their ability to cause ionization. This is shown in figure 1. Particulate radiation can be classified as either light or heavy or whatever changed or unchanged.

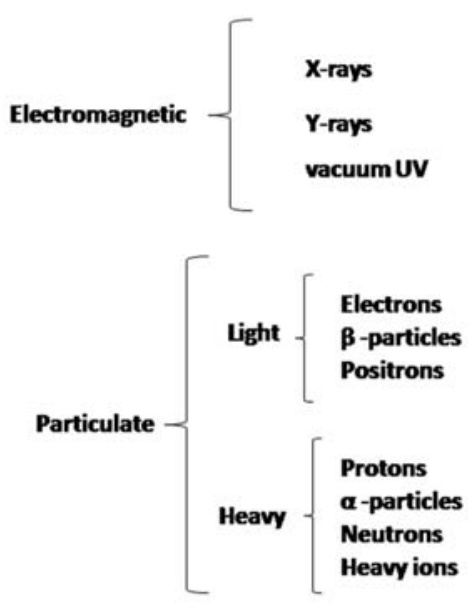

Figure 1: Different ionizing radiations that can affect biological systems. 
While all energies in the ionizing radiation range exert their damaging effect on living cells, not all ranges of non-ionizing radiation have deleterious effects on biological systems; the ultraviolet radiations (UVR) are however, significant for their damaging consequences. Its interaction with biological matter is primarily through the reactions initiated upon excitation in bio-molecules after absorption of these energies. Thus, it can cause effect on these molecules which can absorb the radiation. The visible radiations have as such, no damaging effects; this was also believed to be true for all other longer wavelength radiations (barring the heating effect generated by absorption of such radiations). However, recent findings indicate the possible threats from radio-frequency radiations as well $[5,6]$. The present discussion is restricted to effects of ionizing radiations and only UVR, in the non-ionizing radiation range.

Quantification of the effects of radiation on biological systems required new sets of units [7]. Rather than the unit of exposure dose [roentgen (R)], a unit for the amount of energy actually deposited in the tissue or any biological matter is relevant. Thus, the unit of absorbed dose was introduced in S.I. it is Gray (Gy). 1 Gy is the deposition of 1 Joule of energy in a $1 \mathrm{~kg}$ sample. Biologically, the more realistic unit is the centiGray (cGy) that is equal in value to the traditional unit of absorbed dose, the Rad, which is equal to deposition of 100 ergs/gm.

The effects of the different ionizing radiations though qualitatively similar vary quantitatively to a large extent, like their penetrability, range, energies and so forth. Therefore, there was the need for some normalisation. Dose Equivalentis a measure of the biological effect of radiation that takes into account the type and energy of the radiation as well as how the radiation is distributed [8]. It is measured in sieverts (Sv). $1 \mathrm{~Sv}=1 \mathrm{~Gy} \times \mathrm{Q}$ where $\mathrm{Q}$ is the quality factor, e.g., $\mathrm{Q}=10 \mathrm{for}$ neutrons, thus for $1 \mathrm{~Gy}$ dose, the dose equivalent is $10 \mathrm{~Sv}$. $1 \mathrm{~Sv}$ is a very large dose of radiation which could only happen as a result of a very serious nuclear accident or explosion; generally it is expressed in millisieverts (mSv) or microsieverts $(\mu \mathrm{Sv})$.

The potential biological effects and damages caused by radiation depend on its type and the conditions of the radiation exposure: quality of radiation (linear energy transfer [LET] \& relative biological effectiveness [RBE]), quantity of radiation (received dose of radiation) and exposure conditions (spatial distribution) [7].

Energy deposited per unit track length is known as LET.

$$
\text { LET }=\frac{\mathrm{dE}}{\mathrm{d} x}
$$

Different kinds of radiation have different energy loss effects, or LET. Energy loss effects depend on nature and probability of interaction between radiation particle and body material. To normalize these effects as an empirical parameter the RBE of radiation for producing a given biological effect is introduced.

$$
R B E=\frac{\text { Dose of } 150 \mathrm{~V} \mathrm{X}-\text { rays required to cause effect } x}{\text { Dose of radiation required to cause effect } x}
$$

The RBE of diagnostic x-ray is 1 . Radiations with a lower LET will have a RBE of less than 1. For high LET radiation and the RBE is greater than 1 . As the LET increases, the RBE also increases but a maximum level is reached followed by a reduction due to overkill effect.

The important characteristic of ionizing radiation is the localized release of large amounts of energy. The subsequent events leading to the biological consequences can be divided into four different stages. In the physical stage, the energy is transferred to the irradiated system primarily by photoelectric and Compton's processes which produce ionizations and excitations. The average energy of ionization is $\sim 34 \mathrm{eV}$, which is enough to break a chemical bond (for e.g., $\mathrm{C}=\mathrm{C}$ bond is $4.9 \mathrm{eV}$ ), i.e. cause ionization. The primary species produced through physical interaction are unstable and undergo secondary reaction either spontaneously or by collision with other molecules. This is the physico-chemical stage. It may involve one or more reactions. The free atoms and radicals thus formed then interact with each other and with their environment. This is the chemical stage. If the energy deposition is in the target itself, it is direct action. If the energy deposition is in the neighborhood of the target that ultimately reacts with the target, it is indirect action. As a consequence of alteration in the chemistry of the cellular macromolecules and their altered interactions, there can be changes in the response of the target cells/ organisms. This is the biological stage, the manifestation of these effects can span over an indefinitely long period. Biological effects of radiation result from both direct and indirect action of radiation [9].

The different stages of radiation action and their temporal duration is summarized through a schematic diagram (Figure 2).

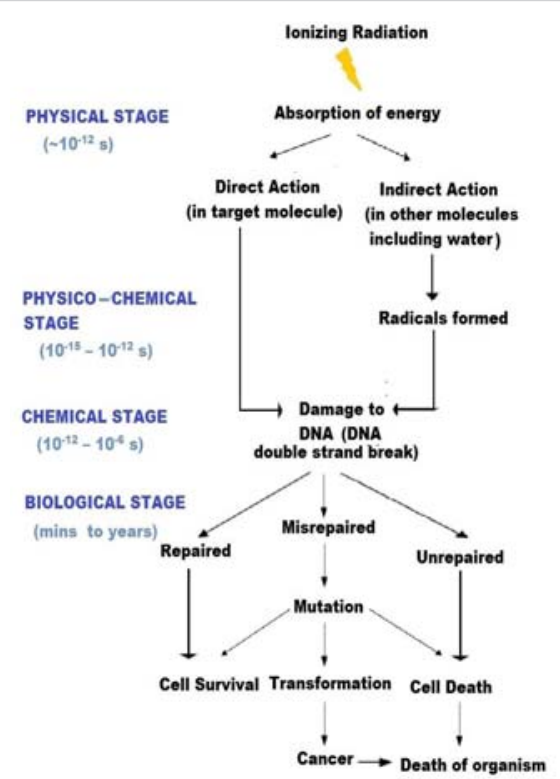

Figure 2: Temporal stages of interaction of radiation with living systems. 
Amongst the different neighboring molecule where energy deposition can significantly affect the target through indirect action is water. Radiolysis of water is a major event [10].

$$
\begin{aligned}
& \mathrm{H}_{2} \mathrm{O} \longrightarrow h v \mathrm{H}_{2} \mathrm{O}+\mathrm{e}^{-} \\
& \mathrm{H}_{2} \mathrm{O}^{+} \longrightarrow \mathrm{H}^{+}+\mathrm{OH} \\
& \mathrm{H}_{2} \mathrm{O}+\mathrm{e}^{-} \longrightarrow \mathrm{H}_{2} \mathrm{O}^{-} \\
& \mathrm{H}_{2} \mathrm{O} \longrightarrow \mathrm{H}^{-} \mathrm{OH}^{-} \\
& \mathrm{OH}+\mathrm{OH} \longrightarrow \mathrm{H}_{2} \mathrm{O}_{2} \\
& \mathrm{e}^{-}+\mathrm{H}_{2} \mathrm{O} \longrightarrow \mathrm{e}_{\text {aq }}^{-} \text {(hydrated electron) }
\end{aligned}
$$

The major radiolytic products of water are $\dot{\mathrm{H}}, \mathrm{OH}$ and $\mathrm{e}_{\mathrm{aq}}^{-}$ $\mathrm{e}_{\text {aq }}^{-}$or hydrated electron has great penetrating effects. It results when an electron ejected due to ionization gets encaged within water molecules. The caging can break at a site that is quite a distance from it's actually site of production. This means that this electron can exert its effect at a site that is remote from where it is produced. For high LET radiations, as the radicals are produced at a close vicinity to each other, the recombined product like $\mathrm{H}_{2} \mathrm{O}_{2}$ is also important for producing oxidative damage. Considering the large percentage of water content in biological samples the contribution from indirect action on target from radiolytic products of water is significant.

The unit of chemical yield for radiation induced reactions is the G-value. $G=1$ indicates that one entity (e.g., one free radical of a given type) is formed or destroyed for each $100 \mathrm{eV}$ of energy absorbed in the medium.

Our knowledge about the biological effects of radiation was initially derived from the effects on microbes. This knowledge was further enhanced from later studies in animal models, cultured cells or data from effects observed on accidentally exposed human subjects. The differential sensitivity of varied organisms has become evident from different experimental observations. Different organisms exhibit disparity in radiation sensitivity $[11,12]$. The relative sensitivity of different organisms based on the dose equivalent that half the number of individuals would survive upon exposure to radiation is shown below for comparison -

Tobacco mosaic virus $2000 \mathrm{sV}$, Amoeba, Insects $1000 \mathrm{sV}$, Snail 200 sV, Bat 150 sV, Bacteria 40 sV, Trout 15 sV, Rat 8 sV, Mouse 5.6 sV, Ape 5.4 sV, Human 3 - 5 sV, Dog 2.6 sV, Pig 2.5 $\mathrm{sV}$, Goat $2.4 \mathrm{sV}$.

The most important macromolecule in cells that is responsible for radiation induced damage to cells / organism is the DNA. DNA damage can result from both direct and indirect effects of radiation. Cytoplasmic changes probably play a minor role in arresting mitosis and inducing cell death; although, swelling of mitochondria and changes in cell wall permeability have been observed. Ionizing radiation is largely responsible for inducing breaks in DNA, although base changes are also possible. If the energy of the radiation is too high, it can cut across both the strands to produce double strand breaks. A large dose of a less energetic radiation can result in induction of a series of single strand breaks. If two such breaks are formed in close vicinity in the two opposite strands, that too, results in double strand break. The extent of DNA damage can be obtained from the following estimate [13] -

Damage/ Gy of X-rays:

40 Double strand breaks

150 DNA cross-links

\section{1,000 Single strand breaks}

\section{2,500 base damages}

Ionizing radiation leads to the release of electron from molecules that result in the generation of ions that leads to break in covalent bond indicating strand breaks in DNA. Radiolysis of water in cells leads to formation $\mathrm{OH}$ radicals that can lead to the generation of reactive oxygen species (ROS) to oxidize proteins and lipids. Generation of abasic sites in DNA, oxidation of the bases and single strand breaks (SSB) are observed. Purine bases are more susceptible to oxidation, particularly guanine. Although several oxidation products are produced in vitro, the formation of 7,8-Dihydro-8-oxo2 '-Deoxyguanosine (8-oxodG) is most abundance and highly mutagenic. It results in $\mathrm{G}$ to $\mathrm{T}$ transversion.

In response to DNA damage cell-cycle check points are activated and there is inhibition in the progression of cellcycle. This allows signaling of DNA repair pathway, however, if extent of damage is large then the cell proceeds towards death most often through apoptosis [14-17].

Different DNA repair processes are involved in repair of DNA damage. While nucleotide excision repairs (NER) is the most general form of repair for any kind of DNA damage. That result in distortion of the double helical structure due to formation of bulky DNA adduct, different DNA glycosylases like 8-oxodG glycosylase, thymine glycol glycosylase, formamidopyrimidine glycosylase can form AP sites in DNA for removal of DNA damaged bases through Base Excision Repair (BER). The DSB that arise from exposure to ionizing radiation can be in two ways- non homologous end joining process (NHEJ), which is responsible for rejoining most of the DSDs, and homologous recombination (HR). NHEJ can rejoin two broken ends of DNA irrespective of the sequence and it is the most often utilized pathway though the fidelity of this process is low. Often there is insertion and/or deletion of nucleotides at the junction leading to mutation. For involvement of HR there is necessity for the presence of homologous strands, as the process requires strand exchange and recombination $[17,18]$. 
Residual DNA damages can result in mutation in genes. The induction of mutations by ionizing radiation was first observed in the fruit fly drosophila. Single gene mutations were studied in human and other cell lines. Generally, it is a linear function of dose, at low doses, 1-10 cGy. In many cases it has been observed that the radiation induced mutation arise due to loss of large segment of the genome, which could be an entire gene or even extend to neighboring loci. Improper rejoining of DNA after repair too, can result in mutation in genes. If there is more than one break, the broken fragments may join in different combinations. The broken ends of the chromosome also possess the ability to join together again after separation resulting in different types of chromosomal aberrations. Chromosomal aberration is an often observed consequence of radiation in mammalian cells. Aberrations like dicentric ring chromosome, fragmentation and large deletion can lead to cytotoxic effects; aneuploidy, translocation and small deletion may not inhibit cell proliferation but is associated in radiation induced carcinogenesis. Neoplastic transformation of normal cells in vitro has been observed on irradiations. A persistent increase in genetic changes has been observed in the progeny of irradiated cells after several generations. This is known as genomic instability. It could be relevant to the induction of malignant tumour formation in irradiated cells [18].

Unrepaired or misrepaired DNA damage may result in cell death. Apoptotic death or programmed cell death can result from irradiation of cells. It is a mechanism of removal of heavily damaged/mutated cells on irradiation. Distinctive changes in cellular morphology, loss of normal structure of nucleus followed by degradation of the DNA that gives the typical 'ladder' in gel are the hallmarks of apoptosis, which is associated with the expression and suppression of several genes involved in regulation of this process. Loss of ability to undergo apoptosis is crucial for tumourogenesis. Although DNA damage is a prime factor in triggering apoptosis in cells, membrane damage and other signaling pathways also contribute to this process [19].

Irradiated cells often undergo irreversible $G_{1} / S$ arrest. $\mathrm{G}_{1} / \mathrm{S}$ arrest is present to allow repair of DNA damage before progression through cell-cycle. At high doses, cells can loose its ability to progress through the cell cycle towards mitosis. Its inability to divide and proliferate results in 'reproductive cell death'. Cellular senescence results from permanent cellcycle arrest induced by radiation senescent cells are more resistant to apoptosis [20].

Radiations in discriminately damages all cellular macromolecules through direct ionization and also from the ROS generated from indirect action. Through DNA lesion are the critical target in cells, damage to proteins are gaining prominence. The toxic effects of the ionizing radiation induced proteins unfolding, chain cleavage, dephosphorylation that leads to loss of functionality and can eventually result in apoptosis.
Cells are equipped with antioxidant enzymes like Superoxide dismutase (SOD), catalase (CAT) and Glutathione Peroxidase (GPx) also present are some small molecule like GSH or vitamins like Vitamin C, Vitamin E and other that can offer protection from oxidative damage. Superoxide $\mathrm{O}_{2}$ is dismutated to $\mathrm{H}_{2} \mathrm{O}_{2}$ and $\mathrm{O}_{2}$ by SOD. Catalase and GPx can enzymatically remove $\mathrm{H}_{2} \mathrm{O}_{2}$ Although oxidative burst from radiation exposure can result in activation of the antioxidant enzymes, some of enzymes can also be inactivated by radiations. Radiation also inactivates the DNA repair enzymes, which can influence the response and induce death in cells [21].

Radiation induces peroxidation of lipid in membrane particularly, the peroxidation of polyunsaturated fatty acids (PUFA). Ionizing radiations attacks the PUFA in lipids. It affects the $\mathrm{C}$-atom between the double bond so that an $\mathrm{H}$ atom is removed and a resonating structure is formed. In presence of oxygen, organic peroxides and peroxy radicals are formed. It leads to alteration in transmembrane processes, disruption of ion transport and increased membrane permeability. Functions of membrane proteins are also affected [21].

The consequences of such processes can lead to different cellular events indicated in the flowchart (Figure 2). The magnitudes of somatic effects of irradiation depend on the following variables: Individual, species, cell types and tissues, extent of exposure (full or partial body) and the total doses [9]

Oxygen plays an important role in modulating radiation sensitivity $[22,23]$. Tissues are more sensitive to radiation if irradiated in oxygenated or aerobic state than in the anoxic (without oxygen) or in hypoxic (low oxygen) condition. This explains the radio-resistance observed in solid tumors; as cells in the core of the tumor are often hypoxic, it presents a challenge for radiotherapy. This characteristic of tissue is described numerically as the oxygen enhancement ratio (OER). The OER is LET dependent [24]. The OER is highest for low LET radiation, having a maximum value of approximately 3 and decreases to 1 for high LET radiation.

Radio-sensitivity also varies with the LET of the radiation [25]. The cellular viability fractions (S) at different doses of irradiation (D) is represented by the following equations -

$\mathrm{S}=\mathrm{e}-^{\alpha \mathrm{D}}$ for high LET radiations

and

$S=e^{-(\alpha D+\beta D \times D)}$ for low LET radiations

Fractionation of dose also modulates radiation induced cell killing [26]. Rather than delivering a single large dose if the same dose of radiation is fractionated in small doses the extent of cell killing is lowered due to sub-lethal damage repair. DNA repair pathways are stimulation in cells between the fractionated doses. During radiation therapy, exposing normal cells in the neighborhood of the tumor to a certain 
extent is unavoidable. Fractionation of dose allows the normal cells some recovery from DNA damage. Most often, cancer cells are not proficient in DNA repair. Defect in DNA repair pathway causes that tumor cells do not recover to the extent of normal cells.

Some other biological factors also influence the sensitivity of cells / tissues. Age is one such factor [27]. Humans are most sensitive before birth. Stem cells are also highly radiosensitive. Radiation sensitivity is high in younger tissues and organs; the sensitivity decreases until maturity so that mature cells are relatively radio-resistant. However, radio-sensitivity again increases in old age. For diagnostic imaging it is important to remember that a fetus is considerably more sensitive to radiation exposure than a child or a mature adult.

Radio-sensitivity ofliving tissuealsovaries withmetabolism $[9,28]$. Highly proliferating cells and tissues with high growth rate have increased radio-sensitivity. However, one of the most notable exceptions to this generalization is the lymphocyte. It is a differentiated cell, not capable of proliferative activity, and yet, it is one of the most radiosensitive cells in the body.

Effect of radiation exposure also depends on whether such exposures are short-term or long-lasting. For e.g., in a medical x-ray the time of exposure is short. The dosage of such exposures is also low. On the other side, short exposures can sometimes also be of a high dose. Radioactive materials that naturally occur in our soil or environment present us with small amounts of radiation that can lead to exposure over the whole lifetime. Therefore, depending on the source of exposure, the dose as well as the time of an exposure can varywhile in some cases, the time may be very short, of the order of seconds or less, in other cases it may occur continuously over our lifetime. Accordingly, radiation effects can be classified in two categories- acute or immediate effects and delayed or chronic (latent) effects. The severity and the time scale for the acute radiation syndrome depend on the maximum dose delivered. The first symptoms generally show up after $6 \mathrm{~h}$. The deterministic effects of chronic exposures are observed after large absorbed doses of radiation. There is usually a threshold dose below which the deterministic effects are not manifested; the severity of the effect increases with increasing dose. Irradiation with ionizing radiation results in a decrease in the white blood cell count (leukopenia). Platelet counts also decreases so that blood clotting is affected to result in bleeding. The dividing cells in the gastrointestinal that are affected on whole body irradiation. The cells have villi to absorb water and electrolytes; their loss can result in diarrhea, which leads to less of elements for survival. Doses as low as $1 \mathrm{~Gy}$ can result in nausea and vomiting. It higher doses above $100 \mathrm{~Gy}$ effect on the central nervous system can lead to disorientation and death. The lethal dose when $50 \%$ of the human irradiated die, called the $\mathrm{LD}_{50}$, is $5 \mathrm{~Gy}$ for men. Some other deterministic effect includes inflammation of throat and other mucous surface, infections, bleeding and epilation. Temporary to permanent sterilely is also observed. The most important stochastic effect of ionizing radiation on human mortality is neoplasia and leukemia [29]. Radiation may be regarded as a two-edged sword; while it cures cancer and it can also be a causative agent for cancer. The probability of its carcinogenic effect increases with dose. There is however, no threshold dose below which the effect will not occur; also, the severity of the effect is independent of the radiation dose. The safe dose of radiation exposure recommended by the international commission on radiological protection (ICRP) and national council on radiation protection and measurements (NCRP) is $50 \mathrm{mSv} /$ year; while the total dose over the life-time should not exceed $50(x-18) m S v$, where $x$ is the age in years [30].

The conventional paradigm for radiation action is that a cell must be hit (targeted) by radiation to exhibit productive responses. Implicit in this notion lies the assumption that radiation affects only those cells where there was absorption of energy by the different cellular structures, particularly in the DNA. Recent findings have revealed that in addition to this, there also exist some non-targeted effects [31]. Radiation causes free radicals to trigger cell-cell communication and cell-matrix communication to cells other than those that are actually "hit" by the radiation. This includes genomic instability and bystander effects. Genomic instability refers to mutations occurring in the progeny of the irradiated cells. On the other hand, bystander effects refer to the responses observed in cells not exposed directly to the radiation, but induced in such cells, through signals emitted by the irradiated cells. Signals are emitted from the cells exposed to direct damage, directly into the external environment through released factors or transferred through gap junctions between adjacent cells. The phenomenon of bystander effect was originally attributed to ionizing radiation. Studies have revealed that bystander effect of ionizing radiation resulted in lowering of cellular survival, induced cytogenetic damage, produced genomic instability, induced mutation, enhanced apoptosis, produced alteration in gene expression pattern, induced chromosomal aberrations and micronuclei formation, and even produced cell transformation [32].

UV light (200-280 nm) that forms a part of the nonionizing range of the spectrum of radiation from the sun, which comprises UVC (200-280 nm), UVB (280-315 nm) and UVA (315-400 nm) [33]. Natural UVC from sunlight is completely observed by the atmosphere, but there are many manmade sources that include germicidal lamp welders lamp and such like. The ozone layer cuts off most of the UVB from the sunlight but about $5 \%$ is still incident on the earth. The UVA range comprises $95 \%$ of the UV ray from the sun that penetrate the atmosphere to reach the earth.

UVC is on the range of wavelength absorbs by DNA; it results in formation pyridine dimmers, particularly thymine dimmers. Two type of dimmers are formed- the cyclobutane type dimmer and the 6-4 photoproduct. Small amount of strand breaks and oxidation product in bases are also formed. 
UVB is responsible for sun burn and tanning. It can result in strand break and oxidation of bases like 8-oxoguanine. UVB is implicated as a prime factor for incidence of cancer from exposure to sunlight. UVA primarily causes oxidative damage. Though, pyrimidine dimmers are also formed, but the relative yield is relatively much low. Formation of sister chromatid exchange is much more for UV irradiation compare to that induced by ionizing radiation [34]. UV radiation can result in DNA-protein crosslinkages. It also interacts with proteins alone to result in less of functions. It can cause unfolding to expose the hydrophobic residues leading to aggregation of proteins. UV radiation also results in oxidation of lipids and alteration in lipid composition. Altered permeability can result in necrotic death in cells [35]. The important effect of irradiation in organisms includes sunburn and premature aging of skin, also known as photoaging. The wrinkle, leathery skin and liver spots are some of the features associated with photoaging. The UVA and UVB range of the solar UV radiation is primarily responsible for these effects. UVR has damaging effect on the eyes; it can lead to cataract formation. Suppression of immune system and formation of melanoma and non-melanoma type of skin cancer are the other significant hazards associated with UV exposure [36].

Although, the types of DNA damages are different; the general downstream effects are similar to ionizing radiation. UV-irradiated cells experiencing DNA damage, exhibit chromosomal aberrations, undergo repair and mutation. Cells unable to repair their DNA damage are killed by necrosis, apoptosis or autophagy and also exhibit alteration in gene expression, though the concerned genes may different; UV radiation is also responsible for induction of skin cancer.

Relative to explorations in bystander effects of ionizing radiations the literature on such effects from different ranges of UV radiations are limited [37]. DNA and membrane damage, generation of ROS, mutation, genomic instability and reduction in cell viability have been observed by some investigators, while contrary findings have also been noted. The responses often depend on wavelength, cell types, proliferation state and its metabolic state. Our laboratory is involved in the studying the bystander effects of UV radiations that revealed that the responses of UVC-bystander cells are conspicuously different. These cells show no change in morphology and viability; there is no DNA or membrane damage but, cells are temporarily arrested at $\mathrm{G}_{2} / \mathrm{M}$ phase and there is induction of enhanced antioxidant enzyme activities [38]. The bystander cells were however, less sensitive on subsequent exposure to a number genotoxic agents including UVC $[38,39]$. Cell killing was less through lowering of necrotic death; but, death through apoptosis and autophagy remained unaffected [40]. For UVA induced bystander effect, the induced resistance was through inhibition of autophagy and apoptosis (unpublished data). Enhanced cellular viability was not due to difference in DNA repair rate but rather due to lowering of ROS production leading to reduction in DNA damage and lipid peroxidation [40]. The lowering of sensitivity to UV radiation in bystander cells was exhibited in these cells only until the time the enhanced antioxidant activities were observed [41]. Inflammatory responses were also lowered [42].

The exact nature of signals for bystander effects and their relative importance are still not clearly known; also unknown is the significance of such effects on health. Such findings however, indicate that the targetsize is greater than the actually irradiated volume. This is important for radiotherapy. Also UVA radiation can be used in conjunction with radiosensitizers for use in photodynamic therapy (PDT) $[43,44]$. Considering the importance of use of radiation for therapeutic applications the question remains - why have they evolved? And whether it is beneficial or detrimental response? The indications from several studies showing the damaging nature of bystander responses for ionizing radiations has aroused concern about the effective amplification of its deleterious effects. In fact, bystander effects of ionizing radiations have been implicated for the relapse of tumors and secondary cancer formation. However, the bystander responses for UV light are quiet distinct. Our findings demonstratedfor the first time that UVC related bystander effect stimulated an intrinsic protective response that is mediated primarily through induction of antioxidant activities. Our recent findings revealed that UVAbystander responses may be a protective response and does not increase the tumorigenic responses (unpublished data). UV radiation most importantly UVA contributes largely the physiological process of extrinsic aging- photoaging [45]. It is though also relevant to expose the possible contribution of bystander cells to the process of photoaging. Further studies using different wavelengths of UV light in different types of cells are necessary to establish the distinctiveness in bystander response between ionizing and non-ionizing radiations.

\section{Acknowledgement}

This work was supported by University of Kalyani, DSTPURSE,GoI and UGC-SAP, GoI, Department of Biochemistry \& Biophysics, University of Kalyani.

\section{References}

1. Assmus A, Early History of X-rays, Summer. 1995: 10-24.

2. Shepherd JA, Ng BK, Fan B, Schwartz AV, Cawthon P, et al. Modeling the shape and composition of the human body using dual energy $X$-ray absorptiometry images. PloS One. 2017; 12: e0175857. PubMed: https://pubmed.ncbi.nlm.nih.gov/28423041/

3. Greenwald HP. Who survives cancer? Univ of California Press. 1992.

4. Lea DE. Actions of radiations on living cells. Actions of radiations on living cells. 1955.

5. Manna D, Ghosh R. Effect of radiofrequency radiation in cultured mammalian cells: A review. Electromagn Biol Med. 2016; 35: 265-301. PubMed: https://pubmed.ncbi.nlm.nih.gov/27053138/

6. Hansda S, Mitra A, Ghosh R. Studies to explore the UVA photosensitizing action of 9-phenylacridine in cells by interaction with DNA. Nucleosides Nucleotides Nucleic Acids. 2021; 1-30. PubMed: https://pubmed.ncbi.nlm.nih.gov/33586599/

7. Seltzer SM, Bartlett DT, Burns DT, Dietze G, Menzel HG, et al Fundamental quantities and units for ionizing radiation. ICRU Journal. 2011; 11: 1 . 
8. Valentin J. Relative biological effectiveness (RBE), quality factor (Q), and radiation weighting factor (wR): ICRP Publication 92. Ann ICRP. 2003; 33: 1-21.

9. Alpen EL. Radiation biophysics. Academic press; 1997.

10. Samuel AH, Magee JL. Theory of radiation chemistry. II. Track effects in radiolysis of water. J Chem Phys. 1953; 21: 1080-1087.

11. Harrison FL, Anderson SL. Taxonomic and developmental aspects of radiosensitivity. Lawrence Livermore National Lab., CA (United States). 1996.

12. Pfafflin JR, Ziegler EN, editors. Encyclopedia of environmental science and engineering. Taylor \& Francis; 1(A-L) fifth edition. 1992.

13. Hall EJ, Giaccia AJ. Molecular mechanisms of DNA and chromosome damage and repair. Radiobiology for the radiologist. 7th ed. Philadelphia: Lippincott Williams \& Wilkins. 2012: 12-34.

14. Friedberg EC, Walker GC, Siede W, Putte PV. DNA repair and mutagenesis. Trends in Biochemical Sciences. 1995; 20: 440.

15. Goodarzi AA, Anikin A, Pearson DD. Environmental sources of ionizing radiation and their health consequences. Genome Stability, Academic Press. 2016; 569-581.

16. Hack, RC. lonizing radiation. Occupational Health Practice. Butterworth-Heinemann, 1989. 151-174.

17. Cannan WJ, Pederson DS. Mechanisms and consequences of doublestrand DNA break formation in chromatin. J Cell Physiol. 2016; 231: 3-14. PubMed: https://pubmed.ncbi.nlm.nih.gov/26040249/

18. Leadon, Steven A. Repair of DNA damage produced by ionizing radiation: A mini review. Semin Radiat Oncol. 1996; 6: 295-305. PubMed: https://pubmed.ncbi.nlm.nih.gov/10717187/

19. Little JB. Principal Cellular and Tissue Effects of Radiation. HollandFrei Cancer Medicine. 6th edition. Hamilton (ON): BC Decker. 2003. PubMed: https://www.ncbi.nlm.nih.gov/books/NBK12344/

20. Li M, You L, Xue J, Lu Y. lonizing radiation-induced cellular senescence in normal, non-transformed cells and the involved DNA damage response: A mini review. Front Pharmacol. 2018; 9: 522.

PubMed: https://pubmed.ncbi.nlm.nih.gov/29872395/

21. Reisz JA, Bansal N, Qian J, Zhao W, Furdui CM. Effects of ionizing radiation on biologicalmolecules-mechanisms ofdamageandemerging methods of detection. Antioxid Redox Signal. 2014; 21: 260-292. PubMed: https://pubmed.ncbi.nlm.nih.gov/24382094/

22. Little JB. Cellular effects of ionizing radiation. New Engl J Med. 1968; 278: 369-376.

PubMed: https://pubmed.ncbi.nlm.nih.gov/4865594/

23. Zhang $\mathrm{H}$, Koch CJ, Wallen CA, Wheeler KT. Radiation-induced DNA damage in tumors and normal tissues. III. Oxygen dependence of the formation of strand breaks and DNA-protein crosslinks. Radiat Res. 1995; 142: 163-168.

PubMed: https://pubmed.ncbi.nlm.nih.gov/7724730/

24. Alper T, Bryant PE. Reduction in oxygen enhancement ratio with increase in LET: Tests of two hypotheses. Int J Radiat Biol Relat Stud Phys Chem Med. 1974; 26: 203-218.

PubMed: https://pubmed.ncbi.nlm.nih.gov/4609938/

25. Barendsen GW. Parameters of linear-quadratic radiation dose-effect relationships: dependence on LET and mechanisms of reproductive cell death. Int J Radiat Bio. 1997; 71: 649-655.

PubMed: https://pubmed.ncbi.nlm.nih.gov/9246179/

26. Barendsen GW. Dose fractionation, dose rate and iso-effect relationships for normal tissue responses. Int J Radiat Oncol Bio Phys. 1982; 8: 1981-1997.

PubMed: https://pubmed.ncbi.nlm.nih.gov/6759484/

27. Joksic G, Petrovic S, llic Z. Age-related changes in radiation-induced micronuclei among healthy adults. Braz J Med Bio Res. 2004; 37:
1111-1117.

PubMed: https://pubmed.ncbi.nlm.nih.gov/15273813/

28. Bushong SC. Radiologic Science for Technologists-E-Book: Physics. Biology, and Protection: Elsevier Health Sciences. 2013.

29. Valentin J. The 2007 recommendations of the international commission on radiological protection. Elsevier; 2008.

30. Cardis E, Gilbert ES, Carpenter L, Howe G, Kato I, et al. Effects of low doses and low dose rates of external ionizing radiation: cancer mortality among nuclear industry workers in three countries. Radiat Res. 1995; 142: 117-132.

PubMed: https://pubmed.ncbi.nlm.nih.gov/7724726/

31. Morgan WF. Non-targeted and delayed effects of exposure to ionizing radiation: I. Radiation-induced genomic instability and bystander effects in vitro. Radiat Res. 2003; 159: 567-580.

32. Baskar R. Emerging role of radiation induced bystander effects: Cell communications and carcinogenesis. Genome integr. 2010; 1: 13.

33. Grossweiner LI, Smith KC. The science of photobiology. Photophysics. 2nd Edition. 1989: 1-46.

34. Kiefer J. Effects of ultraviolet radiation on DNA. In Chromosomal Alterations. Springer, Berlin, Heidelberg. 2007: 39-53.

35. Santos AL, Moreirinha C, Lopes D, Esteves AC, Henriques I, et al Effects of UV radiation on the lipids and proteins of bacteria studied by mid-infrared spectroscopy. Environ Sci Technol. 2013; 47: 6306-6315. PubMed: https://pubmed.ncbi.nlm.nih.gov/23692317/

36. World Health Organization. Health and environmental effects of ultraviolet radiation: a summary of Environmental health criteria 160 , ultraviolet radiation. World Health Organization. 1995.

37. Widel M. Bystander effect induced by UV radiation; why should we be interested? Postepy Hig Med Dosw (Online). 2012; 66: 828-837. PubMed: https://pubmed.ncbi.nlm.nih.gov/23175338/

38. Ghosh R, Guha D, Bhowmik S. UV released factors induce antioxidant defense in A375 cells. Photochem Photobiol. 2012; 88: 708-716. PubMed: https://pubmed.ncbi.nIm.nih.gov/22296560/

39. Ghosh R, Bhaumik G. Supernatant medium from UV-irradiated cells influences the cytotoxicity and mutagenicity of V79 cells. Mutation Research/Environmental Mutagenesis and Related Subjects. 1995; 335: 129-135.

PubMed: https://pubmed.ncbi.nlm.nih.gov/7477043/

40. Ghosh R, Guha D, Bhowmik S, Karmakar S. Antioxidant enzymes and the mechanism of the bystander effect induced by ultraviolet $C$ irradiation of A375 human melanoma cells. Mutation Research/Genetic Toxicology and Environmental Mutagenesis. 2013; 757: 83-90. PubMed: https://pubmed.ncbi.nlm.nih.gov/23845763/

41. Ghosh R, Guha D, Bhowmik S, Karmakar S. Some UV-bystander effects are mediated through induction of antioxidant defense in mammalian cells. Ind J Biochem Biophys. 2012; 49: 371-378. PubMed: https://pubmed.ncbi.nlm.nih.gov/23259324/

42. Guha D, Bhowmik $S$, Ghosh R. Influence of ultraviolet $C$ bystander effect on inflammatory response in $A 375$ cells subsequent exposure to ultraviolet C and hydrogen peroxide. Ind J Biochem Biophys. 2014; 51: 552-558. PubMed: https://pubmed.ncbi.nlm.nih.gov/25823229/

43. Hua H, Cheng J, Bu W, Liu J, Ma W, et al. 5-Aminolevulinic Acid-Based Photodynamic therapy Pretreatment Mitigates Ultraviolet A-Induced Oxidative Photodamage. Oxid Med Cell Longev. 2018; 2018: 9420745. PubMed: https://pubmed.ncbi.nlm.nih.gov/30524664/

44. Hansda S, Ghosh G, Ghosh R. 9-phenyl acridine photosensitizes A375 cells to UVA radiation. Heliyon. 2020; 6: e04733.

PubMed: https://pubmed.ncbi.nlm.nih.gov/32944667/

45. Ghosh R. Role of Proteases in Photo-aging of the Skin. In Proteases in Physiology and Pathology 2017; 435-449. 